\title{
Isochromosomy Yp
}

INSERM

\section{Source}

INSERM. (1999). Orphanet: an online rare disease and orphan drug data base.

Isochromosomy Yp. ORPHA:98797

Isochromosomy Yp is a rare gonosome anomaly characterized by various clinical

presentations including normal healthy fertile males, male phenotype with infertility, and males with ambiguous genitalia or incomplete masculinization. 